\title{
Immunoglobulin G4-Negative Inflammatory Pseudotumors of the Pancreas
}

\author{
Matthew T. Geiselmann ${ }^{\mathrm{a}, \mathrm{j}}$, Daniel J. Acampa ${ }^{\mathrm{a}, \mathrm{j}}$, Joshua Melamed ${ }^{\mathrm{b}}$, Farzana Arif ${ }^{\mathrm{c}}$, \\ Kazuaki Takabe ${ }^{\text {d, e, f, g, h, Eric Seitelman }}{ }^{\text {, }}$, Rajiv Datta ${ }^{b}$, \\ Ganesh Gunasekaran ${ }^{\mathrm{b}, \mathrm{i}}$, Hideo Takahashib, k
}

\begin{abstract}
Inflammatory pseudotumor (IPT) can occur in any organ, but rarely shows pancreatic involvement. While surgical excision has been recommended as the primary treatment for IPT of the pancreas in the past, some authors suggest observation while medical management often results in regression. Corticosteroids, nonsteroidal anti-inflammatory drugs and immunosuppressive therapy have been used to treat IPTs. Spontaneous regression has also been reported in IPT managed without surgical intervention. A 62-year-old female was evaluated for worsening abdominal pain and a mass in the neck of the pancreas that was identified on ultrasound. Further imaging with magnetic resonance imaging revealed a pancreatic mass with dilated pancreatic duct and an atrophic parenchyma of the pancreatic neck. Her serum tumor markers were not elevated. As this lesion appeared to be resectable pancreatic cancer based on cross-sectional imaging, no biopsy was performed prior to surgical resection. Distal pancreatectomy and splenectomy was recommended and the patient desired to proceed. Her recovery was uneventful with no postoperative complications, including pancreatic fistula. Final pathology revealed a lesion consistent with the diagnosis of immunoglobulin G4 (IgG4)-negative IPT without neoplasm. IPT of the pancreas is a difficult entity to diagnose and treat due to clinical and
\end{abstract}

Manuscript submitted November 1, 2021, accepted November 25, 2021

Published online December 8, 2021

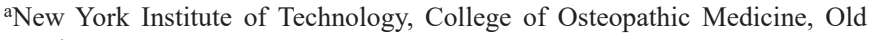
Westbury, NY, USA

bDepartment of Surgery, Mount Sinai South Nassau, Oceanside, NY, USA 'Department of Pathology, Mount Sinai South Nassau, Oceanside, NY, USA

${ }^{\mathrm{d} D e p a r t m e n t}$ of Surgical Oncology, Roswell Park Comprehensive Cancer Center, Buffalo, NY, USA

eDepartment of Surgery, University at Buffalo Jacobs School of Medicine and Biomedical Sciences, the State University of New York, Buffalo, NY, USA

fDepartment of Breast Surgery and Oncology, Tokyo Medical University, Tokyo, Japan

gDepartment of Surgery, Yokohama City University, Yokohama, Japan

hDepartment of Surgery, Niigata University Graduate School of Medical and Dental Sciences, Niigata, Japan

'Department of Surgery, Division of HPB Surgery, Mount Sinai Hospital, New York, NY, USA

${ }^{j}$ Both authors contributed equally.

${ }^{k}$ Corresponding Author: Hideo Takahashi, Department of Surgery, Mount Sinai South Nassau, Oceanside, NY, USA. Email: hideo.takahashi@snch.org

doi: https://doi.org/10.14740/wjon1432 imaging characteristics closely resembling pancreatic adenocarcinoma. Biopsy with immunohistochemical analysis can be useful in diagnosing IPT; however, symptomatic lesions and concerning findings on crosssectional imaging may warrant more definitive surgical intervention.

Keywords: Inflammatory pseudotumor; Pancreas; Normal IgG4

\section{Introduction}

An inflammatory pseudotumor (IPT) is a benign, mass-forming lesion composed of inflammatory infiltrate surrounding myofibroblastic and fibroblastic proliferation [1]. The etiology of IPT is not well understood, but has been associated with chronic inflammatory conditions, trauma, and lymphoproliferative processes [2]. IPT was first described after a tumor was resected from the lung parenchyma of a child by Brunn in 1939 [3]. It was named due to its radiological and clinical similarities to malignant tumors [4]. It has since been observed in nearly every organ in the body, including the pancreas [4$7]$, although it is rather rare in the pancreas [6, 8-11]. Unfortunately, this entity can clinically and radiographically mimic both pancreatic carcinoma and chronic pancreatitis [9]. Previous literature suggests pancreatic IPT in adults is more common in women and that the pancreatic head is most commonly involved ( $60 \%$ of cases) as opposed to the body and tail [12]. About $5-10 \%$ of pancreatectomies performed with the suspicion of malignancy reveal non-malignant IPTs $[2,13,14]$. IPT of the pancreas poses a diagnostic challenge, as it is difficult to rule out malignancy of the pancreas in a safe and accurate fashion prior to resection [2]. Even with negative preoperative biopsy via endoscopic ultrasound, given dismal prognosis of pancreatic cancer, surgical intervention is often recommended for those with reasonable perioperative risks.

We have recently experienced a case of immunoglobulin G4 (IgG4)-negative IPT of the pancreatic neck successfully managed with distal pancreatectomy and splenectomy, and herein present a case report with literature review.

\section{Case Report}

A 62-year-old female without any significant past medical his- 
tory was referred to the surgical oncology department with a newly identified pancreatic neck mass. It was discovered during her workup of worsening right upper quadrant pain that had been present for several years. The pain was intermittent, stabbing, radiating to her mid back, exacerbated by food and associated with nausea, bloating, constipation and loss of appetite. An abdominal ultrasound demonstrated a hypoechoic lesion in the pancreatic body region measuring $2.2 \times 1.8 \times 1.9 \mathrm{~cm}$. Subsequent magnetic resonance imaging (MRI) found a hypoenhancing mass in the pancreatic body which was moderate intensity on T2 sequence, measuring $2.4 \times 1.9 \times 3.2 \mathrm{~cm}$, causing mild upstream dilation of the pancreatic duct in the body and tail (Fig. $1 \mathrm{a}, \mathrm{b})$. The lesion abutted the portal confluence and there was no arterial involvement. Interestingly, there was additional downstream pancreatic ductal dilation up to $4 \mathrm{~mm}$ and the pancreatic neck was atrophic as well. The cross-sectional imaging findings were highly concerning for pancreatic adenocarcinoma. After discussion in a multidisciplinary tumor board, surgical resection without tissue diagnosis was considered reasonable given the tumor location and distinct imaging characteristics. Neoadjuvant chemotherapy with tissue diagnosis was considered but forgone, as a potential tumor growth during the neoadjuvant treatment would possibly make this tumor unresectable. Her serum carcinoembryonic antigen (CEA), carbohydrate antigen 19-9 (CA199) and IgG4 level were within normal limits.

She underwent a diagnostic laparoscopy, and open subtotal distal pancreatectomy and splenectomy. There was no peritoneal disease or liver metastasis on the diagnostic laparoscopy. A firm mass was identified in the inferomedial margin of the pancreatic neck, the body was dissected and the pancreas was elevated from the retroperitoneum. The pancreatic neck was then transected with good margins and complete lymphadenectomy along the celiac axis were performed. A Jackson Pratt drain was placed in the pancreatic bed.

Her postoperative course was overall unremarkable. Her diet was gradually advanced and she was discharged on postoperative day 6 . And the drain was removed in the office.

Pathological evaluation revealed a firm, white, bulky mass near the medial margin measuring $3.5 \times 3.6 \times 2.1 \mathrm{~cm}$. Histologic evaluation showed an intact architecture with a tumefactive-forming chronic pancreatitis. There are exuberant lymphocytic infiltrate and copious multinucleated giant cells involving lobules and periductal regions. There was no evidence of carcinoma in the pancreas specimen as well as in all 16 resected lymph nodes (Fig. 2a, b). While IgG was focally positive on immunohistochemistry (IHC) staining, IgG4 stain was negative (Fig. 3a, b). Also, cytokeratin 7 (CK-7) stain was localized to the epithelial cells (Fig. 4). Immunostain for anaplastic lymphoma kinase-1 (ALK-1) was negative. While autoimmune pancreatitis could not be fully excluded, the lesion is suggestive of an inflammatory pseudotumor without any evidence of carcinoma.

\section{Discussion}

Pancreatic cancer is a calamitous diagnosis, as nearly $94 \%$ of diagnosed cases worldwide resulted in death in 2020. It is the seventh leading cause of cancer death in both males and fe-
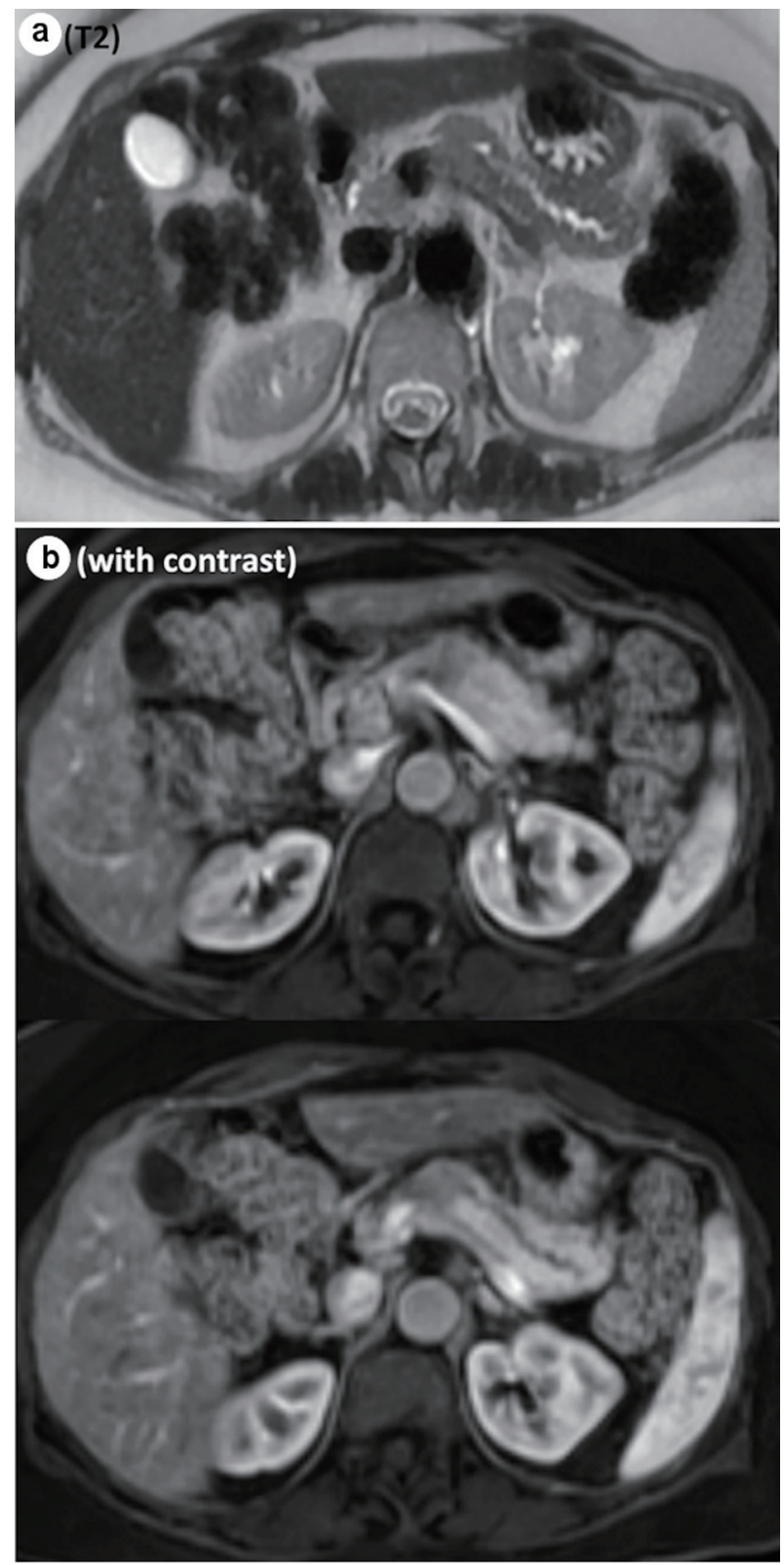

Figure 1. Representative images from MRI. (a) Abrupt cut off of the main pancreatic duct on the T2 sequence. (b) Hypoenhancing mass, measuring $2.4 \times 1.9 \times 3.2 \mathrm{~cm}$, on the contrast study. MRI: magnetic resonance imaging.

males throughout the world, totaling at 466,000 deaths out of 496,000 cases [15]. Although the incidence of other types of cancers, such as breast and colorectal, have declined in recent years, the incidence of pancreatic cancer remains relatively stable. Due to these trends, European studies have predicted pancreatic cancer to surpass breast cancer as the third leading cause of cancer death in Europe by $2025[15,16]$. A solid mass at the pancreatic head/neck has a wide differential diagnosis including but not limited to: adenocarcinoma, neuroendocrine tumor, other rare malignant histologies, chronic mass forming pancreatitis, and IPT $[17,18]$. 


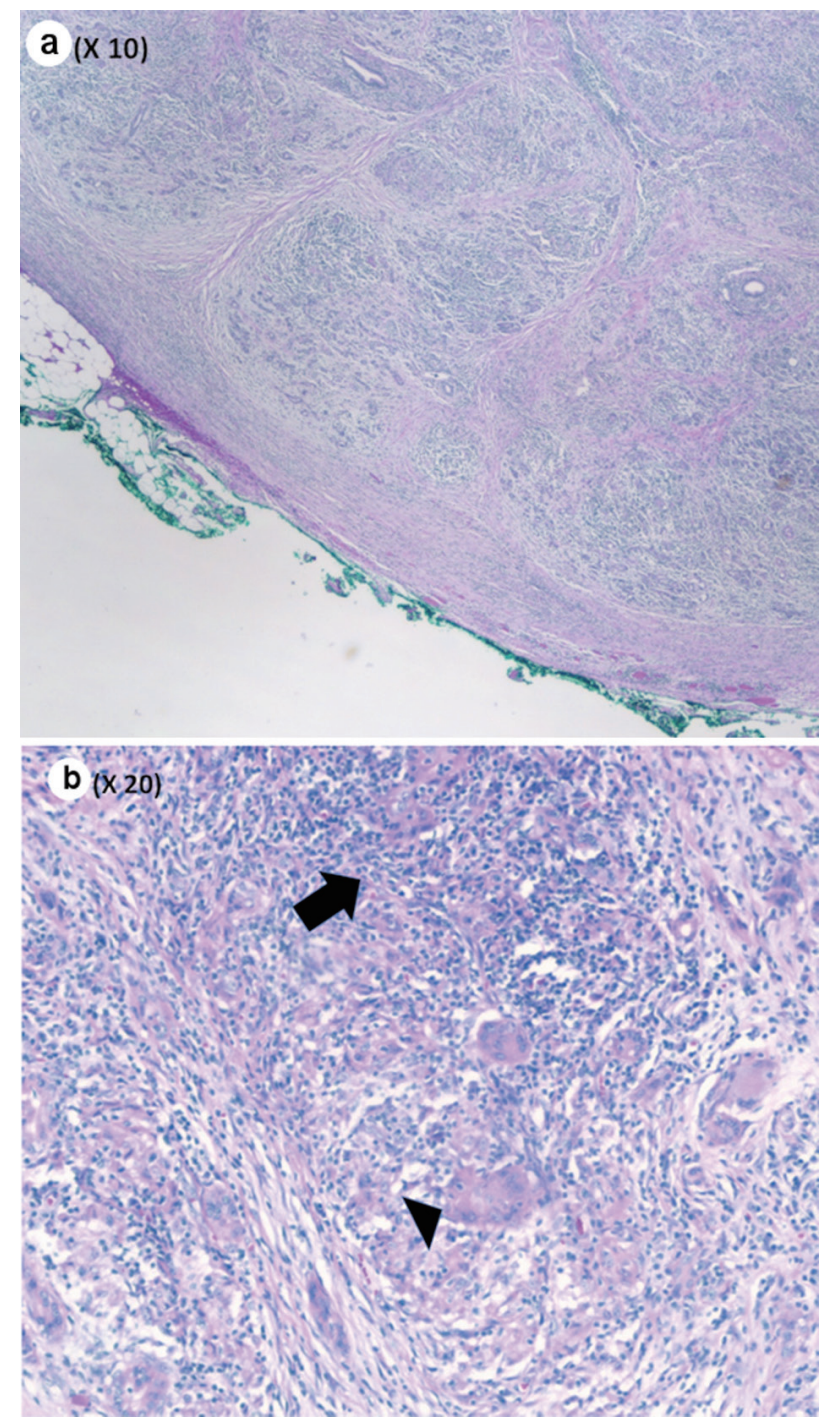

Figure 2. (a) Pancreas parenchyma with intact lobular architecture and exuberant chronic inflammation (low power, $\times 10$ ). (b) Chronic inflammatory infiltrate (black arrow) and giant cell (black arrowhead) (medium power, $\times 20)$.

IPT is described as an uncommon, benign and tumefactive lesion composed of a variety of infiltrative inflammatory and mesenchymal cells. IPT has been defined as a benign mimicker of malignant processes and must be considered in the evaluation of any neoplasm, as successful treatment may be achieved without radical surgical intervention [19]. While the origin of IPT remains unclear, some literature suggests trauma, surgery, and autoimmune mechanisms as possible etiologies [2, 4]. IPT arising from the pancreas is extraordinarily rare, with only a few cases reports in the English literature [2, 20-22]. The radiographic appearance of pancreatic IPT is highly variable, requiring histological evaluation for definitive diagnosis [2]. Contrast CT of intra-abdominal IPT demonstrates a variety of patterns including early peripheral with delayed central filling, heterogenous, homogenous, as well as non-enhancing lesions $[4,23,24]$. MRI demonstrates hypointensity on T1-weighted
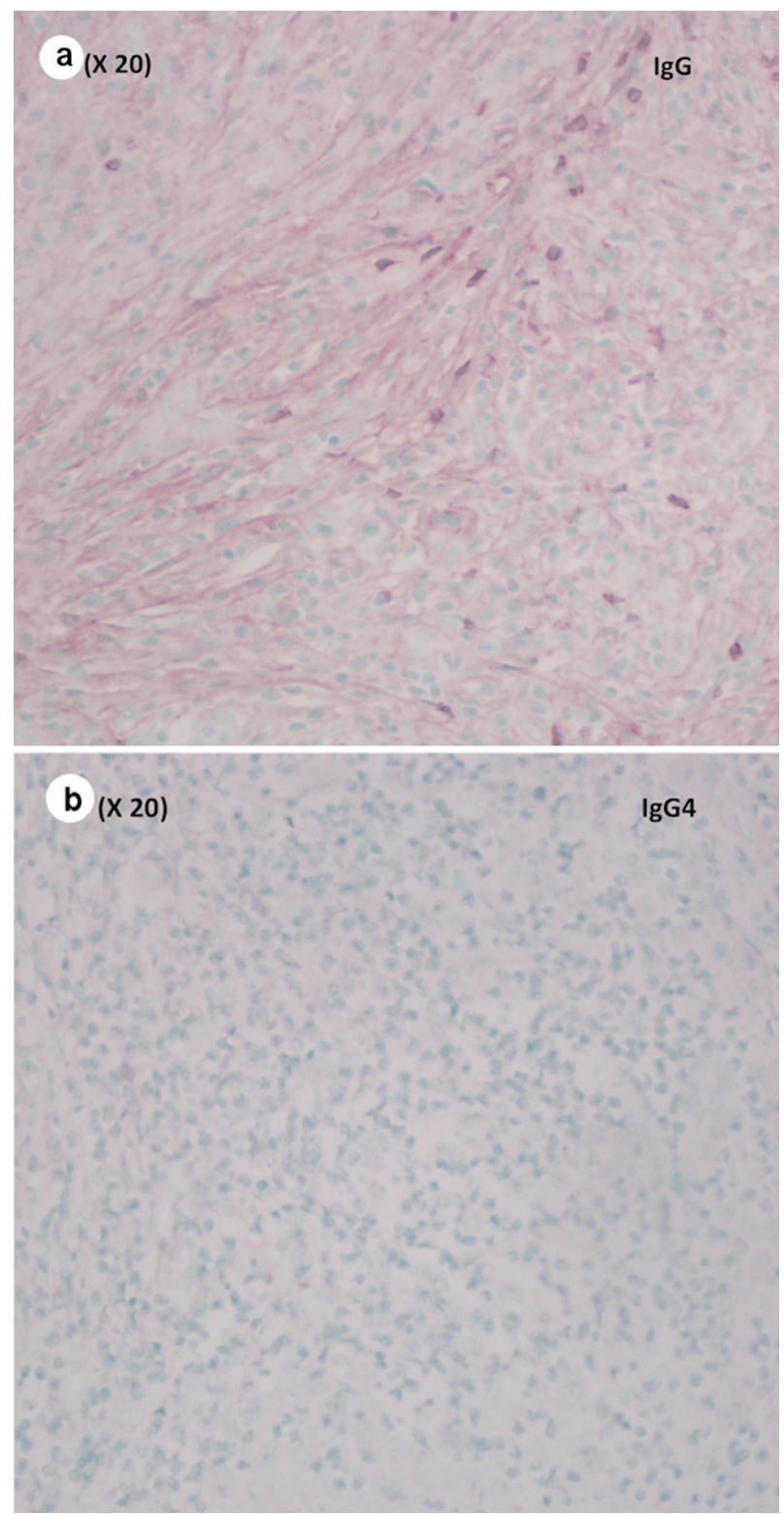

Figure 3. (a) Focally positive immunostain for $\lg G$ (medium power, $\times$ 20). (b) Negative immunostain for IgG4 (medium power, $\times 20$ ). IgG4: immunoglobulin G4.

images [4, 25-27], and hyper- $[4,27]$ or hypointensity [25, 26] on T2-weighted images. However, these highly variable findings cannot distinguish IPT from other conditions such as pancreatic carcinoma and chronic pancreatitis on imaging characteristics alone $[2,4]$.

Similar to our case, Kroft et al described a case of IPT arising in the pancreatic body in a young female. Although this lesion was initially observed several years due to benign findings on needle aspiration of the mass, a subsequent distal pancreatectomy was eventually performed due to significant increase of the tumor size and the patient symptomatology, such as significant weight loss, abdominal pain and microcytic anemia. Following resection, her symptoms were resolved completely, and final 


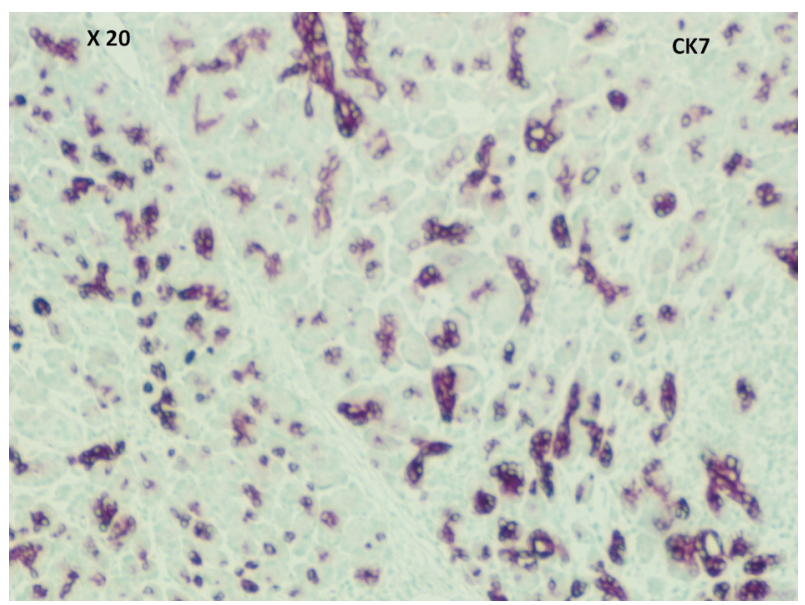

Figure 4. CK-7 was positive in epithelial cells (medium power, $\times 20$ ). CK-7: cytokeratin 7.

pathology demonstrated the diagnosis of IPT with the presence of myofibroblasts [21]. Likewise, DeRubertis and colleagues also reported a similar case in which an elderly female patient presented with a 2-month history of fatigue, nausea, postprandial abdominal pain and vomiting and significant weight loss. Cross-sectional imaging revealed a pancreatic mass in the tail. She underwent laparoscopic distal pancreatectomy as this was suspicious for malignancy. Analysis of intraoperative frozen sections was inconclusive in differentiating between low-grade carcinoma and pancreatitis. Final pathology revealed IPT of the pancreatic tail, and the patient's symptoms completely resolved [20]. These case reports demonstrated that pancreatic IPT could cause significant symptoms despite benign in nature. Due to concerning symptomatology as well as difficulty of differentiating IPT from pancreatic cancer on cross-sectional imaging, curative surgical resection is often required as definitive treatment even the initial biopsy was negative for malignancy.

The histology of IPT is consistent with both acute and chronic inflammatory processes, involving proliferation of lymphocytes, plasma cells, myofibroblastic spindle cells, and fibrous collagen $[19,28,29]$. IPT has been found to be a feature of IgG4-positive sclerosing disease [19]. This condition exhibits IgG4-positive T-cell and plasma cell invasion of various tissues, resulting in chronic pathologies such as autoimmune pancreatitis and sclerosing cholangitis [19,28]. The condition was discovered in 2003 and is currently poorly understood [30]. Upon diagnosis, two of the following three major findings should be present: dense lymphoplasmacytic infiltrate, storiform fibrosis and obliterative phlebitis [31]. While less characteristic findings such as non-necrotizing arteritis and tissue eosinophilia can be present, histopathologic features such as epithelioid cell granulomas and neutrophilic infiltration suggest non-IgG4-related disease [31]. Until 2012, no comprehensive diagnostic criteria had been established for IgG4-related disease. Umehara et al proposed the following criteria to establish the diagnosis of IgG4-related disease: 1) serum IgG4 concentration $>135 \mathrm{mg} / \mathrm{dL}$, and 2) $>40 \%$ of IgG+ plasma cells being IgG4 + and $>10$ cells/high powered field of biopsy sample [32].

Investigating for IgG4-related disease is crucial, as this pathology typically responds very well to steroid therapy [32]. Therefore, by establishing this diagnosis on biopsy, one can potentially avoid the morbidity of resection and treat with steroids. Given the tumor's imaging characteristics and the symptomatic nature of the lesion in our case, we believe that it was in the best interest of the patient to proceed with pancreatectomy despite the possibility of being IPT.

Although pancreatic IPT has been documented as a mass forming lesion in the setting of IgG4-related autoimmune pancreatitis [28], IgG4-positive IPTs have also been seen in the absence of autoimmune pancreatitis [19, 33, 34]. Our patient did not demonstrate any systemic evidence of IgG4-related disease and the IHC stains for IgG4 markers were negative. These findings distinguished this patient's condition from IgG4-related disease. Although IgG4-negative pseudotumors of other organs have been reported [35], to our knowledge, we only found one other case report of IgG4-negative IPT of the pancreas in the English literature [2].

As with any suspicious lesion, a definitive diagnosis is achieved via biopsy and histopathologic analysis. Regarding IPTs of the pancreas, surgical resection is often performed as there are significant concerns for malignancy, which was in our case. Since pancreatic resection portends significant morbidity and possible mortality to the patients, some authors reported their experience of observation with non-operative therapies resulting in regression. Nonsteroidal anti-inflammatory medications and corticosteroids have been previously used to treat IPTs for palliation $[9,32,36]$ or to make the mass a more resectable size and configuration $[6,12,19,37,38]$ in confirmed cases of IPTs. While rare, spontaneous regression of lesions has been reported $[2,19,39-41]$. After successful surgical resection or spontaneously regression, the recurrence of gastrointestinal IPT has been reported in the literature, which is reportedly around $18-40 \%[2$, $19,39]$. Nevertheless, whether surgical resection or non-operative therapy is used, the prognosis for IPT is favorable, although long-term follow-up is necessary to evaluate for recurrence [12].

In conclusion, we presented a rare case of IPT in the pancreas in a 62-year-old female, with vague, worsening abdominal pain. Due to strong suspicion for malignancy on cross-sectional imaging, she underwent distal pancreatectomy and splenectomy without major postoperative complications. Definitive diagnosis required histopathological analysis and IHC.

\section{Acknowledgments}

None to declare.

\section{Financial Disclosure}

Authors have no financial disclosure or funding related to the current study.

\section{Conflict of Interest}

Authors have no conflict of interest. 


\section{Informed Consent}

Informed consent was obtained from the patient.

\section{Author Contributions}

Study concept: MTG, DJA, JM, GG, HT. Writing - draft: MTG, DJA, JM, FA, HT. Writing - review and editing: KT, ES, RD, GG, HT. Supervision: ES, RD, GG, HT.

\section{Data Availability}

The data supporting the findings of this study are available from the corresponding author upon reasonable request.

\section{References}

1. Yamamoto H, Watanabe K, Nagata M, Tasaki K, Honda I, Watanabe S, Soda H, et al. Inflammatory myofibroblastic tumor (IMT) of the pancreas. J Hepatobiliary Pancreat Surg. 2002;9(1):116-119.

2. Baiao JM, Martins RM, Correia JG, Jordao D, Vieira Caroco T, Caetano Oliveira R, Agostinho PG, et al. Inflammatory Pseudotumor of the Pancreas Mimicking a Pancreatic Neoplasm. Case Rep Gastroenterol. 2019;13(2):245-252.

3. Brunn H. Two interesting benign lung tumors of contradictory histopathology: remarks on the necessity for maintaining the chest tumor registry. Journal of Thoracic Surgery. 1939;9(2):119-131.

4. Narla LD, Newman B, Spottswood SS, Narla S, Kolli R. Inflammatory pseudotumor. Radiographics. 2003;23(3):719-729.

5. Mehrabani S, Shokri-Shirvani J. A rare inflammatory myofibroblastic tumor of pancreas in a child with cholestasis presentation. Acta Medica Iranica. 2020;58(7):358-361.

6. Jones EC, Clement PB, Young RH. Inflammatory pseudotumor of the urinary bladder. A clinicopathological, immunohistochemical, ultrastructural, and flow cytometric study of 13 cases. Am J Surg Pathol. 1993;17(3):264274.

7. Umiker WO, Iverson L. Postinflammatory tumors of the lung; report of four cases simulating xanthoma, fibroma, or plasma cell tumor. J Thorac Surg. 1954;28(1):55-63.

8. Battal M, Kartal K, Tuncel D, Bostanci O. Inflammatory myofibroblastic pancreas tumor: a case report. Clin Case Rep. 2016;4(12):1122-1124.

9. Panda D, Mukhopadhyay D, Datta C, Chattopadhyay BK, Chatterjee U, Shinde R. Inflammatory myofibroblastic tumor arising in the pancreatic head: a rare case report. Indian J Surg. 2015;77(6):538-540.

10. Lobo FD, Sinha R, Pai RR, Prabhu S. Inflammatory myofibroblastic tumor of the pancreas: a rare entity. Indian J Pathol Microbiol. 2010;53(3):585-587.

11. Pungpapong S, Geiger XJ, Raimondo M. Inflamma- tory myofibroblastic tumor presenting as a pancreatic mass: a case report and review of the literature. JOP. 2004;5(5):360-367.

12. Ding $\mathrm{D}, \mathrm{Bu} \mathrm{X}$, Tian $\mathrm{F}$. Inflammatory myofibroblastic tumor in the head of the pancreas with anorexia and vomiting in a 69-year-old man: A case report. Oncol Lett. 2016;12(2):1546-1550.

13. Lalwani N, Mannelli L, Ganeshan DM, Shanbhogue AK, Dighe MK, Tiwari HA, Maximin S, et al. Uncommon pancreatic tumors and pseudotumors. Abdom Imaging. 2015;40(1):167-180.

14. Adsay NV, Basturk O, Klimstra DS, Kloppel G. Pancreatic pseudotumors: non-neoplastic solid lesions of the pancreas that clinically mimic pancreas cancer. Semin Diagn Pathol. 2004;21(4):260-267.

15. Sung H, Ferlay J, Siegel RL, Laversanne M, Soerjomataram I, Jemal A, Bray F. Global cancer statistics 2020: GLOBOCAN Estimates of incidence and mortality worldwide for 36 cancers in 185 countries. CA Cancer J Clin. 2021;71(3):209-249.

16. Ferlay J, Partensky C, Bray F. More deaths from pancreatic cancer than breast cancer in the EU by 2017. Acta Oncol. 2016;55(9-10):1158-1160.

17. Ryan DP, Hong TS, Bardeesy N. Pancreatic adenocarcinoma. N Engl J Med. 2014;371(11):1039-1049.

18. Ryu DH, Sung RH, Kang MH, Choi JW. Lymphoepithelial cyst of the pancreas mimicking malignant cystic tumor: report of a case. Korean J Hepatobiliary Pancreat Surg. 2015;19(3):129-132.

19. Patnana M, Sevrukov AB, Elsayes KM, Viswanathan $\mathrm{C}$, Lubner $\mathrm{M}$, Menias CO. Inflammatory pseudotumor: the great mimicker. AJR Am J Roentgenol. 2012;198(3):W217-227.

20. DeRubertis BG, McGinty J, Rivera M, Miskovitz PF, Fahey TJ, 3rd. Laparoscopic distal pancreatectomy for inflammatory pseudotumor of the pancreas. Surg Endosc. 2004;18(6):1001.

21. Kroft SH, Stryker SJ, Winter JN, Ergun G, Rao MS. Inflammatory pseudotumor of the pancreas. Int J Pancreatol. 1995;18(3):277-283.

22. Dudiak KM. Abdominal case of the day. Inflammatory pseudotumor of the pancreas. AJR Am J Roentgenol. 1993;160(6):1324-1325.

23. Levy S, Sauvanet A, Diebold MD, Marcus C, Da Costa N, Thiefin G. Spontaneous regression of an inflammatory pseudotumor of the liver presenting as an obstructing malignant biliary tumor. Gastrointest Endosc. 2001;53(3):371-374.

24. Slavotinek JP, Bourne AJ, Sage MR, Freeman JK. Inflammatory pseudotumour of the pancreas in a child. Pediatr Radiol. 2000;30(11):801-803.

25. Flisak ME, Budris DM, Olson MC, Zarling EJ. Inflammatory pseudotumor of the liver: appearance on MRI. Clin Imaging. 1994;18(1):1-3.

26. Materne R, Van Beers BE, Gigot JF, Horsmans Y, Lacrosse M, Pringot J. Inflammatory pseudotumor of the liver: MRI with mangafodipir trisodium. J Comput Assist Tomogr. 1998;22(1):82-84.

27. Torzilli G, Inoue K, Midorikawa Y, Hui AM, Takayama 
T, Makuuchi M. Inflammatory pseudotumors of the liver: prevalence and clinical impact in surgical patients. Hepatogastroenterology. 2001;48(40):1118-1123.

28. Yamamoto H, Yamaguchi H, Aishima S, Oda Y, Kohashi $\mathrm{K}$, Oshiro Y, Tsuneyoshi M. Inflammatory myofibroblastic tumor versus IgG4-related sclerosing disease and inflammatory pseudotumor: a comparative clinicopathologic study. Am J Surg Pathol. 2009;33(9):1330-1340.

29. Kwak JW, Paik CN, Jung SH, Chang UI, Lee KM, Chung WC, Yoo JY, et al. An inflammatory myofibroblastic tumor of the ampulla of vater successfully managed with endoscopic papillectomy: report of a case. Gut Liver. 2010;4(3):419-422.

30. Reyes JVM, Maldonado D, Stern AS, Brown M. A case of IgG4-related kidney disease developing while on steroid treatment for autoimmune IgG4 pancreatitis. J Investig Med High Impact Case Rep. 2021;9:23247096211026500.

31. Lang D, Zwerina J, Pieringer H. IgG4-related disease: current challenges and future prospects. Ther Clin Risk Manag. 2016;12:189-199.

32. Umehara H, Okazaki K, Masaki Y, Kawano M, Yamamoto M, Saeki T, Matsui S, et al. Comprehensive diagnostic criteria for IgG4-related disease (IgG4-RD), 2011. Mod Rheumatol. 2012;22(1):21-30.

33. Kamisawa T, Takuma K, Egawa N, Tsuruta K, Sasaki T. Autoimmune pancreatitis and IgG4-related sclerosing disease. Nat Rev Gastroenterol Hepatol. 2010;7(7):401409.
34. Notohara K, Arimoto M, Wani Y, Fujisawa M. Autoimmune pancreatitis: pancreatic manifestation of IgG4-related disease. Pathol Case Rev. 2010;15:219-224.

35. Almajali F, Almajali M, Schwetye K, Alexopoulos G, Khan M, Coppens J, Mercier P. IgG4-negative pituitary inflammatory pseudotumor with sphenoidal involvement resembling a macroadenoma. Br J Neurosurg. 2020:1-7.

36. Ng SH, Tay JS, Lai EL. IgG4-related disease: an atypical presentation of steroid-responsive renal mass. BMJ Case Rep. 2021;14(5):bcr-2020-240611.

37. Sim A, Lee MW, Nguyen GK. Inflammatory myofibroblastic tumour of the pancreas. Can J Surg. 2008;51(1):E23-24.

38. Dagash H, Koh C, Cohen M, Sprigg A, Walker J. Inflammatory myofibroblastic tumor of the pancreas: a case report of 2 pediatric cases - steroids or surgery? J Pediatr Surg. 2009;44(9):1839-1841.

39. Sanders BM, West KW, Gingalewski C, Engum S, Davis M, Grosfeld JL. Inflammatory pseudotumor of the alimentary tract: clinical and surgical experience. J Pediatr Surg. 2001;36(1):169-173.

40. Tang L, Lai EC, Cong WM, Li AJ, Fu SY, Pan ZY, Zhou WP, et al. Inflammatory myofibroblastic tumor of the liver: a cohort study. World J Surg. 2010;34(2):309-313.

41. Yamaguchi J, Sakamoto Y, Sano T, Shimada K, Kosuge T. Spontaneous regression of inflammatory pseudotumor of the liver: report of three cases. Surg Today. 2007;37(6):525-529. 